\title{
Perspectivas feministas sobre (in)justicias ocupacionales de maternidades adolescentes
}

\author{
Perspectivas feministas sobre (in) justiças ocupacionais de maternidades \\ para adolescentes
}

\section{Feminist perspectives on occupational (in)justices of adolescent motherhood}

\author{
Paula Mesa Molina ${ }^{a}$ (D), Pía Rodríguez-Garrido ${ }^{b}$ (D), Juan Andrés Pino-Morán ${ }^{c}$ (D) \\ ${ }^{a}$ Escola Universitària d'Infermeria i Teràpia Ocupacional - EUIT, Terrassa, España. \\ ${ }^{\text {b}}$ Facultad de Ciencias, Universidad Mayor, Santiago, Chile. Dirección de Equidad de Género y Diversidades, \\ Universidad de O'Higgins-UOH, Rancagua, Chile. \\ 'Escuela de Salud, Carrera Terapia Ocupacional, Universidad de O’Higgins - UOH, Rancagua, Chile. Escola \\ Universitària d’Infermeria i Teràpia Ocupacional - EUIT, Terrassa, España.
}

Cómo citar: Molina, P. M., Rodríguez-Garrido, P., \& Pino-Morán, J. A. (2021). Perspectivas feministas sobre (in)justicias ocupacionales de maternidades adolescentes. Cadernos Brasileiros de Terapia Ocupacional, 29, e2869. https://doi.org/10.1590/2526-8910.ctoAO2169

\begin{abstract}
Resumen
Introducción: La maternidad supone un importante desafío ocupacional para las mujeres, situación que se complejiza cuando son adolescentes. Objetivo: Analizar las trayectorias de mujeres madres adolescentes desde la perspectiva de la Terapia Ocupacional Feminista. Método: Estudio cualitativo que, a través de entrevistas en profundidad y un análisis temático, analizó seis experiencias de maternidades adolescentes en España. Resultados: Emergieron tres categorías centrales de análisis que explican parte de las trayectorias ocupacionales. Destacamos dentro de éstas: la "Precarización ocupacional: una vulnerabilidad sexo-genérica", la cual da cuenta de las condicionantes previas que posibilitan la gestación adolescente; “Una ocupación familiar: maternidad (inter)dependiente”, refleja la organización familiar de los cuidados y la crianza dando cuenta de la relevancia de las figuras femeninas que sostienen esta experiencia; y, finalmente, la categoría "El giro ocupacional: sus repercusiones psicosociales", que considera el impacto de la maternidad en las proyecciones, tanto a nivel educativo como en su inserción laboral. Conclusión: Las trayectorias de las mujeres madres adolescentes reflejan un vaivén de emociones y complejidades en sus maternidades asociadas al periodo de vida en el cual la llevan a cabo. Por ello, la emergencia de una Terapia Ocupacional Feminista consciente de las inequidades de género permite interpelar la injusticia ocupacional que enfrentan las mujeres madres adolescentes por una vida más justa y digna.
\end{abstract}


Palabras-clave: Maternidad, Adolescencia, Género, Justicia Social, Terapia Ocupacional, Feminismo.

\section{$\underline{\text { Resumo }}$}

Introduçáo: A maternidade é um importante desafio ocupacional para as mulheres, situação que se torna mais complexa na adolescência. Objetivo: Analisar as trajetórias de mães adolescentes na perspectiva da terapia ocupacional feminista. Método: Estudo qualitativo que, por meio de entrevistas em profundidade e análise temática, analisou seis experiências de maternidades para adolescentes na Espanha. Resultados: emergiram três categorias centrais de análise que explicam parte das trajetórias ocupacionais. Dentre elas, destacamos a "Precariedade ocupacional: uma vulnerabilidade gênero-sexo", que dá conta das condiçôes anteriores que possibilitam a gravidez na adolescência. A categoria "Uma ocupação familiar: maternidade (inter) dependente" reflete a organizaçáo familiar do cuidado e da educaçáo, dando conta da relevância das figuras femininas que sustentam essa experiência. Por fim, a categoria "O turno ocupacional: suas repercussóes psicossociais", considera o impacto da maternidade nas projeções, tanto no nível educacional como na sua inserção laboral. Conclusão: As trajetórias de mães adolescentes refletem uma flutuação de emoçóes e complexidades em suas maternidades associadas ao período da vida em que a realizam. Por isso, o surgimento de uma terapia ocupacional feminista atenta às desigualdades de gênero nos permite desafiar as injustiças ocupacionais enfrentadas pelas mães adolescentes na luta por uma vida mais justa e digna.

Palavras-chave: Maternidade, Adolescência, Gênero, Justiça Social, Terapia Ocupacional, Feminismo.

\section{$\underline{\text { Abstract }}$}

Introduction: Motherhood is an important occupational challenge for women, a situation that becomes more complex when they are adolescents. Objective: To analyze the trajectories of adolescent mothers from the perspective of Feminist Occupational Therapy. Method: Qualitative study that, through in-depth interviews and thematic analysis, analyzed six adolescent maternal experiences in Spain. Results: Three central categories of analysis emerged that explain part of the occupational trajectories. We highlight within them, the "Occupational precariousness: a gender-sex vulnerability", which accounts for the previous conditions that make adolescent pregnancy possible. The category "A family occupation: (inter) dependent motherhood" reflects the family organization of care and upbringing, giving an account of the relevance of the female figures who support this experience. Finally, the category "The occupational shift: its psychosocial repercussions", considers the impact of motherhood in the projections, both at the educational level and in its labor insertion. Conclusion: The trajectories of adolescent mothers reflect a fluctuation of emotions and complexities in their maternity wards associated with the period of life in which they carry it out. Therefore, the emergence of a Feminist Occupational Therapy aware of gender inequalities allows us to challenge the occupational injustice faced by adolescent mothers for a more just and dignified life.

Keywords: Maternity, Adolescence, Gender, Social Justice, Occupational Therapy, Feminism. 


\section{Introducción}

La adolescencia es un periodo complejo de transición entre la niñez y la adultez; conlleva cambios físicos, sociales, sexuales, cognitivos y emocionales que pueden ocasionar conflictos identitarios durante su desarrollo (Allen \& Waterman, 2019). En ese sentido, la adolescencia supone un proceso de generización (Freixas Farré, 1995), que, de acuerdo con Timoneda (2005, p. 66) implica "[...] la construcción psicológica, social y cultural de las características consideradas femeninas o masculinas que se atribuyen a los miembros de cada sexo". En esta etapa, las y los adolescentes emprenden toda una experimentación y búsqueda de lo que será su identidad personal y social, por lo que es el momento en que se perciben como una unidad propia y diferente de las otras personas (Arias et al., 2011). Sin embargo, este contexto en ocasiones se ve alterado en circunstancias como la maternidad no planificada.

La maternidad en mujeres adolescentes es una situación que ha estado presente en todas las sociedades y en los diferentes contextos geográficos. No obstante, y a pesar de ello, no fue abordada como asunto de salud pública, por lo que no se realizaba ningún tipo de intervención para identificar sus condicionantes e implicaciones psicosociales (Hernández \& Gentile, 2018). Recientemente, a partir de los años sesenta, los Estados tomaron conciencia de esta realidad social generando políticas que apuntasen a disminuir estadísticamente la brecha social que esto genera (Hoffman, 1998). La preocupación por llevar a cabo la maternidad en este periodo, está asociada a la presencia de riesgos biopsicosociales (García-Odio \& González, 2018; Loredo-Abdalá et al., 2017) que afectan la salud de las mujeres al momento de la gestación, puerperio y crianza. Estas consecuencias ocurren principalmente por la inmadurez física, fisiológica y psicoemocional propias del ciclo vital que vive la adolescente (Daguerre \& Nativel, 2006).

Desde un enfoque epidemiológico, España tiene una tasa de embarazo adolescente de 9 casos por cada mil (The Save the Children Fund, 2016). Según estadísticas de la Organización Mundial de la Salud (OMS), la tendencia de gestaciones en mujeres adolescentes es decreciente desde 1990, no obstante, se evidencia que un $11 \%$ de nacimientos en todo el mundo son producidos por mujeres adolescentes de entre 15 a 19 años (Organización Mundial de la Salud, 2018). Asimismo, la entidad revela que existe una prevalencia de adolescentes que, a causa de ser mujeres madres de forma temprana, presentan consecuencias psicosociales que afectan directamente en su desarrollo (Organización Mundial de la Salud, 2018). Las causas de aquello se asocian a la poca o nula educación afectiva, sexual y reproductiva que reciben en espacios escolares y al interior de sus hogares (Ganji et al., 2018).

\section{Maternidad(es) y Adolescencia(s): Una Aproximación Desde la Terapia Ocupacional Feminista}

La maternidad es uno de los ámbitos que genera mayor debate y cuestionamiento en los movimientos feministas actualmente (Calquín-Donoso \& Yáñez-Urbina, 2020; Sibrian-Díaz, 2021; Vivas, 2019). Su preocupación revela las precarias condiciones en las cuales se aborda la salud sexual y reproductiva de las mujeres, por parte de toda una 
institucionalidad que da cuenta de una forma particular de ejercicio en clave biopolítica (Foucault, 2009).

Desde las distintas veredas analíticas que el movimiento vindicó -y sigue vindicando-, la maternidad ha sido una de las trincheras fundamentales para interpelar enérgicamente el mandato ejercido hacia las mujeres por el pensamiento heterosexual reflejado en la heteronorma (Wittig, 1992). En ese sentido, la reproducción humana es un hito ocupacional, cultural y político, que ha sido históricamente producida como una forma de explotación naturalizada para las mujeres en las sociedades capitalistas. Sobre esto, autoras como Hannah Arendt y más recientemente Silvia Federici, han sido enfáticas en señalar que "[...] toda la esfera de las actividades centrales para la reproducción de la vida, el trabajo doméstico, la sexualidad y la procreación [...]" (Federici, 2018, p. 13) han sido históricamente atribuidas a la condición humana mujer. Con mayor detalle, Arendt señala que las labores inherentes a las mujeres son propias a estas en tanto se han sociabilizado como "[...] actividad correspondiente al proceso biológico del cuerpo humano [...]" (Arendt, 1958, p. 21).

Lo anterior, es el reflejo de una sociedad que vulnera e invisibiliza la maternidad. Este escenario genera en las corporeidades de las mujeres una completa reestructuración de los cuidados en todas las aristas de sus vidas cotidianas (Gilligan, 2013). En ese sentido, ponemos énfasis en las maternidades que han visto intersectado su ejercicio en tanto las relaciones de poder que la precarizan se articulan como redes fangosas impidiéndoles realizar sus ocupaciones dignamente, es decir, llevar a cabo maternidades que contemplen el apoyo de la comunidad, de las instituciones, de las políticas públicas $\mathrm{y}$ de todo un entorno.

Lo señalado implica visualizar los complejos ejes que atraviesan la maternidad adolescente. Así, la edad, el género, la propia maternidad, la inmadurez tanto física como psicológica, la condición socioeconómica y la escolaridad, son algunas de las cuestiones de gran injerencia de cara al ejercicio libre de la maternidad.

Vélez \& Figueredo (2015) ejemplifican esta situación a través de la repentina transición de la adolescencia a la adultez que las jóvenes vivencian. Este cambio se considera complejo, ya que puede afectar considerablemente su equilibrio ocupacional, limitando y perjudicando así, su calidad de vida.

En ese sentido, la Terapia Ocupacional Feminista (Grandón, 2019; Morrison \& Araya, 2018; Sanz, 2012; Vidal et al., 2017) es una perspectiva emancipadora de cara a problematizar y romper con los estereotipos estigmatizadores de género y edadistas, ya que considera que las tensiones que viven las mujeres madres adolescentes derivan -entre otras cuestiones- de las desigualdades de género y de poder que existen en la sociedad heteropatriarcal, así como el rol adjudicado a la mujer como principal cuidadora, siendo casi inexistente la total corresponsabilidad en la dedicación a la gestación y crianza. De este modo, se devela una alienación ocupacional (Moruno \& Fernández, 2012) generada por un modelo sexista y patriarcal. Por el contrario, la emergencia de una Terapia Ocupacional Feminista como perspectiva epistemológica, nos entrega insumos para repensar y orientar políticas que tengan como propósito la prevención de situaciones de injusticia ocupacional que afecten a las diferentes áreas de desempeño ocupacional, en este caso, de las mujeres madres adolescentes.

En ese sentido, seguimos la propuesta de lograr una justicia ocupacional, entendida esta como "[...] el reconocimiento y la atención a las necesidades ocupacionales de los 
individuos y las comunidades como parte de una sociedad justa y empoderada [...]" (Wilcock \& Townsend, 2000, p. 84) para lograr un horizonte ético y justo de transformación ocupacional (Hocking, 2020).

Así, a partir de lo señalado, el objetivo de esta investigación fue analizar las trayectorias de mujeres madres adolescentes desde la perspectiva de la Terapia Ocupacional Feminista.

\section{Metodología}

\section{Diseño de estudio}

El presente estudio se encuadra en una metodología cualitativa, ya que busca profundizar en las representaciones y significados de las mujeres madres adolescentes, en tanto sus experiencias y relatos generan una aproximación situada e integral del fenómeno de estudio (Valles, 2002). Se optó por este abordaje, ya que permitió acercarnos a la realidad que viven las mujeres madres adolescentes a través de sus trayectorias ocupacionales. Asimismo, fue posible explorar e identificar la información recogida desde un posicionamiento ocupacional feminista (Sanz, 2012) y crítico (Guajardo, 2012), en tanto los problemas y necesidades ocupacionales se contemplaron como posibles factores de riesgo psicosociales que condicionaron e influyeron en el desarrollo vital de las mujeres adolescentes y en su rol de madres expresado en sus trayectorias ocupacionales. El ejericio constante de atender a las narrativas partiendo de premisas sustanciales como las relaciones de poder, las desigualdades de género, las representaciones sociales de la maternidad adolescente y la desigualdad socioeconómica por mencionar algunas, fueron instancias que permitieron aproximarnos a la realidad y a su análisis, desde una posición reflexiva y crítica a la hora de interpretar la información obtenida.

\section{Contexto del estudio}

Se trata de un estudio desarrollado en la ciudad de Terrassa (Barcelona), cuya capital ocupa el $2^{\circ}$ lugar en el ranking de las comunidades más pobladas de España (Cano, 2019). No obstante, las estadísticas muestran un relevante descenso en las tasas de gestación en mujeres adolescentes en los últimos años (Espanha, 2020).

\section{Aproximación a las participantes}

Debido a la imposibilidad para acceder al colectivo mediante al diagnóstico e intervención en un centro de atención de adolescentes, se contactó a través de redes sociales invitando a participar del estudio a mujeres que hubiesen sido madres adolescentes y que quisieran compartir su experiencia. Se utilizó el método de muestreo por bola de nieve (Martín-Crespo \& Salamanca, 2007) con el cual las propias mujeres propusieron y facilitaron la participación de otras mujeres conocidas que habían vivido la misma o similar situación. 


\section{Criterios de selección de las participantes}

El principal criterio de selección consideró las experiencias de mujeres que hubiesen sido madres adolescentes en una edad comprendida entre los 14 y 19 ańos, que hablaran español y que fueran capaces de tomar decisiones de forma autónoma, es decir, que no presentaran dificultades de tipo cognitivas al momento de la entrevista.

La muestra finalmente estuvo compuesta por seis mujeres residentes en Terrassa (Barcelona) procedentes de diferentes países y ciudades (Tabla 1).

Tabla 1. Características de las participantes del estudio.

\begin{tabular}{ccccc}
\hline Nombre ficticio & Edad actual & Nacionalidad & Apoyo familiar & Pareja \\
\hline Nassira (N) & 50 & Marroquí & Presente & Ausente \\
\hline Diana (D) & 26 & Ecuatoriana & Presente & Presente \\
\hline Michel (MI) & 20 & Mexicana & Presente & Ausente \\
\hline Marta (MA) & 27 & Española & Presente & Ausente \\
\hline Sonia (S) & 34 & Española & Presente & Ausente \\
\hline Gimena (G) & 34 & Argentina & Presente & Presente \\
\hline
\end{tabular}

Tabla de elaboración propia.

\section{Técnica de recogida de la información}

La técnica utilizada consistió en la realización de entrevistas en profundidad (Robles, 2011) cuyo objetivo se basó en explorar y conocer aquellos aspectos de la historia ocupacional de mujeres que fueron madres durante su adolescencia, junto a los escenarios sociales y cotidianos que les rodearon.

Las entrevistas se administraron únicamente en formato online de manera gradual, es decir, en distintas instancias por cada entrevistada (Tabla 2). Esto con el objetivo de evitar la sobrecarga mental y emocional que implica la virtualidad, por el mismo motivo que se les propuso responder a través del formato audio, ello con el propósito de considerar el carácter emocional que implicaba narrar ciertos momentos y experiencias de vida fundamentales en el desarrollo de sus maternidades adolescentes y que pudiesen ser percibidos a través de los audios.

Este material fue consignado como nota de campo por las/os autores y posteriormente incluido en el análisis de la información.

Tabla 2. Sesiones de entrevistas online.

\begin{tabular}{cc}
\hline Nombre ficticio & Sesiones \\
\hline Nassira (N) & 7 \\
\hline Diana (D) & 7 \\
\hline Michel (MI) & 7 \\
\hline Marta (MA) & 5 \\
\hline Sonia (S) & 2 \\
\hline Gimena (G) & 4 \\
\hline
\end{tabular}

Tabla de elaboración propia. 


\section{Consideraciones éticas}

Tras contactar con las participantes se llevó a cabo un proceso informativo en el que se les explicó acerca de los criterios que implicaba participar de forma autónoma y legal en la investigación. En ese sentido, y para poder hacer uso de la información extraída, se procedió a firmar el consentimiento informado por parte de las participantes. Este procedimiento fue realizado de forma virtual, debido a la complejidad referente al acceso físico con éstas y a la escasa disponibilidad de tiempo que presentaban.

El manejo de la información y el acercamiento a las participantes se mantuvo siempre bajo los estándares mínimos éticos requeridos en una investigación cualitativa que interactúa y dialoga con personas. De esta manera, se resguardó en todo momento la privacidad, integridad y anonimato de las entrevistadas durante su participación en el estudio.

\section{Rigor metodológico}

Durante todo el proceso de investigación y para la elaboración del manuscrito final, se utilizó la guía para investigaciones cualitativas "Standards for Reporting Qualitative Research" (O’Brien et al., 2014).

\section{Análisis de los datos}

Para el análisis de la información obtenida en las entrevistas y las consideraciones de las notas de campo, se realizó un análisis temático cualitativo de la información.

En primera instancia, se llevó a cabo una lectura en profundidad de las transcripciones del material recogido para luego iniciar su proceso de codificación abierta y categorización (Corbin \& Strauss, 2008).

Posteriormente, la interpretación y el análisis de la información se llevó a cabo mediante el método de comparación constante (Dye et al., 2000). Este consiste en realizar un análisis en el que se combina la codificación de las categorías que surgen de la recogida de información con una comparación simultánea de todos los códigos que se identifican. Asimismo, se realizó de forma paralela una comparación entre las diferentes categorías, cuyo proceso permitió descubrir las relaciones entre los diferentes fenómenos que se observaron, así también, conocer la realidad de la problemática que se presentaba desde la visión de la Terapia Ocupacional Feminista (Sanz, 2012).

\section{Resultados}

A continuación, se presentan los resultados de forma gráfica y descriptiva de acuerdo a las diferentes categorías consideradas relevantes en la selección de la información (Figura 1). 


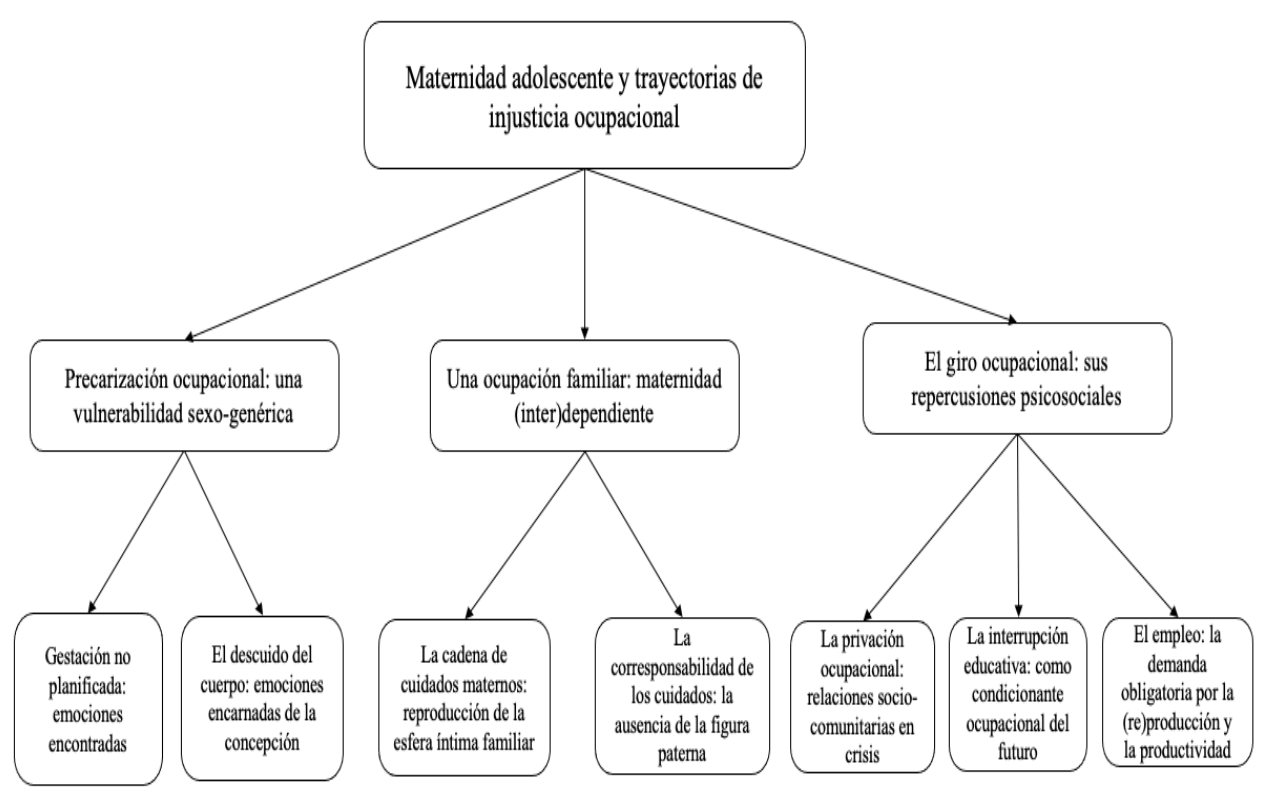

Figura 1. Categorías y subcategorías de análisis. Figura de elaboración propia.

\section{Precarización ocupacional: una vulnerabilidad sexo-genérica}

Cuando analizamos las situaciones personales previas a la gestación adolescente, identificamos varios factores que, desde el punto de vista de las autoras/es, pueden considerarse como desencadenantes de la maternidad en esta etapa. Según la información recogida a través de las vivencias de las mujeres entrevistadas, se devela la existencia de las siguientes situaciones estructurales: ausencia de familia estable, vacío emocional tras el fallecimiento de un familiar y adolescencia desafiante o en rebeldía por problemas sociales y/o económicos. No obstante, cabe destacar que las situaciones mencionadas se viven de una manera particular cuando se es mujer adolescente, ya que muestra en toda su magnitud la vulnerabilidad sexo-genérica de sus ocupaciones y trayectorias.

\section{Gestación no planificada: emociones encontradas}

La información recogida muestra que ninguna de las experiencias analizadas se trató de una gestación planificada. No obstante, todas las participantes dieron continuidad a sus gestaciones. En ese sentido, al momento de preguntarles sobre su reacción inicial ante el conocimiento de la gestación, la mayoría coincide en que sintieron felicidad e ilusión por la noticia, reconociendo a la vez, la falta de madurez y conciencia que tenían sobre la maternidad. Así lo señalan estas participantes:

Pues mira, cuando me enteré de que estuve embarazada del primero, reaccioné bien, me sentí contenta... iPero claro yo tenía 16 años y yo no sabia nada de la vida y estaba enamorada y entonces pues dije... ay que bien! Yo con 16 años 
pensaba que me podia comer el mundo y que ya era grande y podía hacer lo que me daba la gana y no... (D, 2020).

No, no fue planeado, pero cuando supe de su llegada... Jamás pensé en abortarlo $o$ en darlo en adopción. ;Desde que supe que estaba embarazada le di muchisimo amor y hasta la fecha siempre digo que es lo mejor que me ha pasado! (MI, 2020).

Estas experiencias reflejan algunas de las emociones que puede generar la gestación teniendo en cuenta la diversidad de dicho proceso y la particularidad de cada caso, inclusive, sin considerar las transformaciones que se desarrollarán en el maternaje o los cuidados posteriores. Las emociones que reflejan las participantes implican, por un lado, la felicidad que sintieron al recibir la noticia de su gestación, y por otro lado, la dificultad que ello comprendía de cara a la no planificación y a la edad en la cual estaba sucediendo.

\section{El descuido del cuerpo: emociones encarnadas de la concepción}

La representación dominante de la maternidad genera una ilusión vinculada a los mandatos de feminidad como una culminación de un proceso natural para la mujer. Sin embargo, en las adolescentes se generan contradicciones producto de las condiciones materiales y simbólicas de su ciclo vital, donde el temor y la alegría se apoderan de sus subjetividades, generando un vaivén de emociones y significados. En ese sentido, las entrevistadas refirieron sentirse descontentas con el cambio de rutina, así como también, insatisfacción en el desempeño del área del autocuidado al cual otorgaban gran importancia, por lo que manifestaron un importante abandono en el cuidado personal durante la crianza, acompańado de una autopercepción de imagen corporal desfavorable. Así lo describe esta participante:

En el autocuidado cuando estuve embarazada y cuando di a luz fue malo porque yo no me cuidaba, o sea, yo ya... me daba igual como si salía a la calle con moño... me daba igual... El descuido del autocuidado pues fue ese, no arreglarme, no comprarme cosas para mí, comer lo que sea... fue más o menos asi. Además, yo, por ejemplo, descansar no descansaba, descansaba muy poco por el niño... (D, 2020).

En estos casos, la mayoría de las mujeres entrevistadas cuando refirieron pasar por estos difíciles momentos, reconocieron la importante labor que cumplió su familia y entorno en el cuidado y apoyo. De esta manera, se vuelve fundamental la contención familiar a través de favorecer espacios y actividades de autocuidado que le permitan a las mujeres madres adolescentes realizar las actividades de la vida diaria que realizaban previo a su gestación.

\section{Una ocupación familiar: maternidad (inter)dependiente}

La maternidad en la adolescencia y en la cultura occidental, particularmente, supone una posición de dependencia que involucra a todo el núcleo familiar quienes deben acompañar en los cuidados y crianza del recién nacido (Ibarra, 2003). En el caso del grupo estudiado, se observa que es preferentemente la abuela -materna- quien por 
excelencia ocupa un lugar central en la organización de los cuidados familiares. Situación que otorga contención y apoyo a las mujeres durante el proceso de gestación y crianza.

\section{La cadena de cuidados maternos: reproducción de la esfera intima familiar}

Tanto la madurez en desarrollo propia de la etapa adolescente como los condicionantes socio-estructurales son determinantes que dificultan llevar a cabo el ejercicio libre y responsable de la maternidad. Es por ello, por lo que las entrevistadas señalan como fundamental la presencia de familiares que acompañen y apoyen los cuidados de sus hijos/as, así como algunas de ellas destacan la involucración directa de éstos en la economía durante la crianza, mientras que otras resaltan como necesaria la ayuda de los familiares para posibilitar su desarrollo y participación laboral. Así lo describe esta participante:

Cuando quedé embarazada me ayudó mi abuela, bueno siempre estaba la abuela ahi para todo, pudiese o no siempre, estaba ahi dándome ánimos (N, 2020).

Del mismo modo, refieren imprescindible el apoyo económico que el entorno familiar les proporcionó. Tal como lo señala la siguiente participante:

Mi madre, mi hermana...la familia de mi marido, pues como a cualquier embarazada... me ayudaron en el sentido de que pues me escuchaban o las dudas que yo tenía pues me las aclaraban y tal... En el sentido económico mi padre me ayudó mucho emm... y mi marido pues igual (D, 2020).

Con estas experiencias se puede apreciar la generización familiar de los cuidados, cómo se transita por apoyos preferentemente femeninos y cómo las figuras masculinas ocupan la función de provisión económica, consolidando los roles de género y sus ocupaciones.

Además, en la mayoría de los casos -inicialmente-, hubo una reacción molesta y decepcionante del entorno familiar próximo de la mujer adolescente ante el estado de gestación, así como especulaciones negativas acerca de los pensamientos de los demás a causa del estigma y los prejuicios de la sociedad. De acuerdo a las narrativas, se observa la existencia de conflictos ante la gestación. Una de las participantes refiere:

En parte mi familia, toda mi familia hablaba de mi de que era tan chiquita y embarazada y que iqué iba a hacer con mi vida! ...y que si iba a seguir estudiando...y cosas asi, entonces si fue más dificil en ese aspecto porque jamás estuvieron al pendiente de mi (MA, 2020).

Con esto, podemos apreciar la relevancia que tiene la familia y las redes sociales comunitarias en el apoyo y sostén en las maternidades adolescentes, sobre todo y con especial énfasis, cuando no se cuenta con el apoyo y presencia de la figura paterna. 


\section{La corresponsabilidad de los cuidados: la ausencia de la figura paterna}

Las mujeres entrevistadas coinciden en su totalidad en la ausencia de la corresponsabilidad de la figura paterna en los cuidados del recién nacido al momento de asumir la crianza: separación y/o abandono por parte de la pareja, ausencia del apoyo por parte del padre biológico del recién nacido, ideología y comportamiento machista influyente en la participación de la pareja en los cuidados, responsabilidad total por parte de la mujer adolescente en la crianza de su hijo/a, inmadurez y miedo por parte del padre biológico tras la situación y evitación de la adquisición del nuevo rol como padre. Son algunos de los escenarios que refieren haber experimentado las entrevistadas, particularmente, así lo expresan estas participantes:

El padre no se hacía responsable porque sólo le traía la comida y cosas y ya está, pero cuidarla sólo yo le cuidaba, la duchaba, la cambiaba y le daba todo... pero el padre no, qué va, el padre es muy machista decía que era la mujer quien tiene que cuidar a los hijos y eso es lo que me ha pasado a mi (N, 2020).

El papá de mi hijo me ayudó hasta el año, 3-4 meses entonces la responsabilidad de Ian era más para mi que para él porque mi hijo vivía aquí conmigo y su papá lo veía sábados o domingos, entonces yo era la que si estaba malo yo batallaba en las noches y cosas así... Él me ayudaba económicamente y me daba cuando él podia, no siempre. Asi que si... la responsabilidad siempre fue para mi. Y pues si, yo creo que si hay mucha diferencia en mi caso, que los papás están separados (MI, 2020).

Los relatos de las entrevistadas no sólo denotan la precarización en materia de cuidados y responsabilidades afectivas, económicas y sociales para con sus hijos/as, sino, además, revela la cruel normalización por parte de estas al asumir que la responsabilidad que debe adoptar la figura paterna, en este caso, se refleja en la "ayuda" o "colaboración" que este presta en la casa o en los cuidados. La normalización de ese tipo de ayuda por parte de la figura paterna es uno de los motivos por los cuales los movimientos feministas dirigen su lucha hacia la demanda de una ética de cuidados, responsabilidades afectivas, así como de la valoración de la maternidad. Asimismo, se sostiene una lucha en el debate de lo reproductivo/productivo, así como de lo público/privado y de las condiciones genéricas que devienen de estas cuestiones y que por ańos ha relegado el rol de la mujer a espacios de cuidado infra y subvalorados por la sociedad (Amoroso et al., 2003).

\section{El giro ocupacional: sus repercusiones psicosociales}

La crianza muestra un impacto ocupacional en la rutina y en el desempeño ocupacional de las entrevistadas, lo que conlleva un importante cambio en comparación con la rutina diaria que mantenían con anterioridad al nacimiento de sus hijos/as. La mayoría de las informantes coinciden y refieren que esta se trataba de una rutina típica de la adolescencia con un favorable equilibrio entre la productividad (estudios), las actividades significativas de ocio y tiempo libre, junto a su variadas relaciones y vínculos sociales. Desde el nacimiento de sus hijos/as, las mujeres relatan que su rutina se enfocó en la crianza, la productividad y las tareas domésticas, así como refieren presentar rutinas 
interminables y establecer horarios según las demandas de los hijos/as. Por ello, se observa una ausencia relevante de ocupaciones significativas en el día a día, lo que provoca un desequilibrio ocupacional en estas. Por otro lado, destacan la pérdida del rol como adolescente para ocupar el nuevo rol de madre.

\section{La privación ocupacional: relaciones socio-comunitarias en crisis}

Es relevante exponer la información obtenida acerca de las relaciones sociales del colectivo durante la crianza, puesto que se han identificado los siguientes problemas en la mayor parte de las entrevistadas: pérdida o ausencia de amistades y limitación en la participación en ocupaciones significativas relacionadas con la vida social, gran importancia y baja satisfacción en cuanto a las relaciones sociales durante la crianza, presencia del apoyo por parte de amistades durante la gestación - el cual se pierde tras el nacimiento y la crianza del infante -, vivencia de estigmas y críticas negativas por parte de miembros de la familia y personas externas hacia la mujer adolescente por su maternidad. Así lo relata la siguiente participante:

Cuando di a luz y tenía 16-17 años, en la vida social, la gente con la que yo quedaba y salia y todo, pues ya nadie volvió a escribirme... (D, 2020).

Son pocos los casos en los que las participantes declaran disponer de apoyo por parte de amistades. Por lo que se refiere al ambiente ocupacional durante la crianza, las mujeres confiesan presentar una sensación de pérdida de la adolescencia a causa de la limitación que presentan en la participación de actividades significativas e interacciones sociales, a la misma vez que manifiestan sentir tristeza y ańoranza por el abandono de la rutina anterior al nacimiento de su hijo/a. En las narrativas señalan que no se pueden permitir dedicar tiempo a su ocio y a actividades significativas a diario. Como lo señala esta participante:

O sea, yo seguí pensando en querer seguir teniendo la vida de antes, pero... pero no, cuando me di cuenta de que no la iba a poder tener pues... me sentía muy triste (D, 2020).

La información recopilada demuestra que no es frecuente que el colectivo reciba atención profesional tras el nacimiento de sus hijos/as, por ende, relatan una falta de apoyo psicológico, así como también, comentan que la atención sanitaria recibida ha sido únicamente por parte del profesional pediatra que atiende el desarrollo del infante.

Hubiera querido apoyo de otro profesional, más que sociosanitario emm... psicológico porque yo me quedé como con depresión post parto y todo eso yo creo que lo superé sola porque no... O sea, lo tenía muy para mí y ya está. Pero no, no tuve ninguna ayuda (D, 2020).

Las participantes relatan la necesidad de recibir atención psicológica, sobre todo en el periodo post-parto, ya que se reconoce socialmente que el profesional idóneo para otorgar apoyo biopsicosocial en esos momentos son los profesionales psi de cara a su conocimiento en patologías como la depresión postparto. Sin embargo, se desconoce el 
aporte que las y los profesionales Terapeutas Ocupacionales puedan realizar en ese periodo.

\section{La intermupción educativa: una condicionante ocupacional del futuro}

De igual modo que en la actividad laboral se observa la necesidad, y por lo tanto, la dependencia que las mujeres presentaron en relación con los recursos y/o el apoyo familiar como cuidadores. Las participantes refirieron precisar de dicho apoyo durante el horario de estudio con tal de poder compaginar la crianza y favorecer su participación en el proceso educativo. Así lo mencionan estas participantes:

Mi primera hija la tuve con 16 años, en ese momento estaba estudiando y trabajando. Y el segundo lo tuve con 18 y ya no estaba estudiando, estaba trabajando porque tenía una responsabilidad que era la niña (G, 2020).

Mi nivel de estudios pues bueno... (ríe) Un PQPI [Programa de Cualificación Profesional Inicial], y luego pues eso solamente un PQPI y quiero retomarlo, pero bueno en eso estoy mientras que trabajo pues estoy estudiando administración online para poder estar con mi hija también (MA, 2020).

Con estas experiencias, se puede identificar las necesidades que precisan respecto del apoyo por parte de otras personas para el cuidado de sus hijos/as a la hora de compaginar la crianza y sus estudios y/o vida laboral. Se observa que la mayoría de las mujeres han dispuesto de la ayuda de sus familiares próximos con mayor frecuencia por parte de las progenitoras de éstas. No obstante, pese a disponer de apoyo familiar, se identifican problemas para poder desarrollarse favorablemente en el ámbito educacional y/o laboral. Esto es debido a los escasos recursos y apoyos que tienen para poder dejar al infante durante las horas que dedican a la productividad, ya que precisan un horario flexible y/o una adaptación del método de estudio o empleo, con tal de evitar la sobrecarga por parte del familiar cuidador, cumplir con la asistencia, así como cubrir las necesidades básicas del infante e invertir el tiempo deseado con este/a.

\section{El empleo: la demanda obligatoria por la (re)producción y la productividad}

Las situaciones y los problemas que se identifican en el área de la productividad corresponden a la dificultad en la obtención de empleo con horario flexible y compatible con la crianza, vida laboral inestable y cambiante, y casos en los que las mujeres se encuentran ante una situación de inactividad laboral o trabajo a media jornada. Se recalca que las mujeres otorgan gran importancia a la ocupación laboral con tal de lograr desarrollarse de forma autónoma tanto a nivel personal como económico. No obstante, las narrativas de las mujeres entrevistadas demuestran una dependencia económica inicialmente -de sus progenitores para cubrir tanto las necesidades básicas de ellas como la de sus hijos/as. Asimismo, algunas de las mujeres entrevistadas refieren haber tenido faltas de asistencia laborales por motivos del cuidado de sus hijos/as, debido a la incompatibilidad y/o falta de recursos y apoyo. En ese sentido, manifiestan justificar dichas faltas por motivos personales, ocultando la realidad por temor e inseguridad a ser 
despedidas a causa de las responsabilidades que conlleva el rol de madre. Ejemplo de esto último, lo describe la siguiente participante:

Yo aprendi a no decir... o sea, a no poner de excusa a mis hijos porque sabia que me iba a crear un problema en mi trabajo y creándomelo a mi... se los creo a ellos. Porque claro, imaginate... si me echan del trabajo no tengo cómo mantenerlos a ellos, o sea que era porque se tenía que hacer asi (D, 2020).

Las extensas y agotadoras rutinas de vida que llevan las mujeres entrevistadas no pueden sino afectarles en su desempeño y equilibrio ocupacional. Tal como lo señala la siguiente participante:

Ahora mi dia a día es levantarme a las 6:30, hacer la comida para que se la lleve mi marido al trabajo, levantar a las 7:30 a los niños para que se duchen, que se cambien, desayunen, llevarlos al cole... Después de las 9 irme a trabajar algunas horas que tenga que me salgan, porque me van saliendo horas de limpieza (D, 2020).

Impresiona que, a pesar de las intensas luchas y demandas por parte de los movimientos feministas y diversos colectivos sociales que demandan una vida digna y justa, las mujeres tengan que "decidir" si criar, desarrollarse profesionalmente o vincularse afectivamente, ya que todas estas variables juntas, terminan provocando el fenómeno observable de la "doble presencia" (Estevan-Reina et al., 2014), en tanto la responsabilidad total que recae en las mujeres producto de la necesidad de responder y dedicarse al ámbito laboral, familiar y de cuidados, viéndose obligadas a sacrificar la participación en actividades significativas de diferentes áreas (Amoroso et al., 2003).

Este desequilibrio ocupacional, pone de manifiesto la necesidad de fortalecer la Terapia Ocupacional Feminista, que profundice sus intervenciones e investigaciones desde un enfoque situado, político y de género. Esto, permite fomentar la participación equilibrada en ocupaciones significativas interpelando el patrón heteronormado, reduciendo y/o eliminando las injusticias ocupacionales derivadas de dicho orden.

\section{Discusión}

Las trayectorias ocupacionales de las mujeres madres adolescentes develan una posición estructural particular por donde circulan sus experiencias vitales que ratifican los factores que precarizaron sus procesos de gestación y crianza. En ese sentido, los hallazgos señalados en la "Precarización ocupacional: una vulnerabilidad sexo-genérica" expresan la importancia de las variables socio culturales y cómo estas afectan el desarrollo de la maternidad para las mujeres. Así, se mencionan características internas como el complejo periodo vital en el cual se desarrolla la maternidad, y características externas vinculadas a la ausencia de un núcleo familiar estable que acompañe el proceso de gestación y crianza principalmente asociado a la figura paterna o de padre biológico del recién nacido, no así del rol que ocupó -en la mayoría de los casos- la abuela o madre de la adolescente. Autores como Henríquez-Valencia (2016) señala que, junto a las características mencionadas, el capital socioeconómico se ańade como una condicionante que agudiza los factores de riesgo y por ende complejiza llevar a cabo la 
maternidad, sobre todo, si el nivel socioeconómico de la mujer que gesta es medio-bajo o bajo.

Situación que se torna más complicada aún cuando es una "Gestación no planificada: emociones encontradas", pues rara vez se encuentran casos de mujeres adolescentes que deseen planificar una gestación en este periodo de vida, no obstante, en escenarios de pobreza extrema se vuelve un distractor de la realidad de cara a darle sentido a sus vidas ante un entorno adverso (García, 2014). Sin embargo, en este estudio, las entrevistadas refieren no haber planificado sus gestaciones, a pesar de mostrarse ilusionadas por la noticia. En ese sentido, Rojas \& Méndez (2016) señalan que la inmadurez propia de la adolescencia en ocasiones no permite dilucidar con claridad el complejo panorama al cual se enfrentarán, por ende, les hace sentir emociones positivas de su gestación sin dimensionar otras variables. En concordancia, las entrevistadas refieren haber vivido una madurez repentina con la noticia de la gestación, situación expresada en la subcategoría "El descuido del cuerpo: emociones encarnadas de la concepción". La madurez repentina que señalan se traduce en el rol socio cultural aprendido por las mujeres y que las vincula directamente con la maternidad, sobre esto, Chodorow (1984) identifica que las mujeres se transforman en madres en tanto son socializadas como tal desde pequeñas a partir de interacciones familiares a lo largo de su desarrollo. De este modo, para el entorno familiar la maternidad es una dimensión inculcada indirectamente desde la infancia en las adolescentes. En ese sentido, la categoría "Ocupación familiar: maternidad (inter)dependiente", se entiende como la trama que articula la socialización de la maternidad en el entorno familiar y el apoyo otorgado a la mujer madre adolescente, aunque en ocasiones esto último no suceda debido a circunstancias de distanciamiento y/o quiebre familiar, tal como lo señala Diniz et al. (2012).

En este sentido, el apoyo del entorno familiar se refleja en la figura de la madre de la adolescente, situación expresada en la subcategoría "La cadena de cuidados maternos: reproducción de la esfera íntima familiar". Los hallazgos de este estudio identifican sentimientos ambiguos desde la madre hacia la adolescente que transitan desde la molestia y enojo por la gestación propiamente tal, al apoyo y contención.

Por su parte, Gilligan (2013) señala que las labores de cuidados han estado históricamente generizadas. A esta situación la autora le atribuye que las labores relacionadas a lo reproductivo y su visualización femenina implicó que se internalizara con mayor fuerza esta comprensión, ello explicaría, en parte, el motivo por el cual las madres de las adolescentes tenderían a hacerse cargo de sus nietos/as como si fuesen sus propios hijos/as y no así los padres de las adolescentes gestantes, situación que se tornaría más amena si la figura del padre o acompañante de la adolescente fuese más activa.

En la subcategoría "La corresponsabilidad de los cuidados: la ausencia de la figura paterna" se devela una irresponsabilidad por parte del padre biológico en materia afectiva, económica y de cuidados. Las mujeres madres adolescentes deben lidiar con la gestación y crianza de manera precaria y en ocasiones con la nula intervención de la figura paterna de sus hijos/as. Este acontecimiento se da no sólo en las maternidades adolescentes. Como explica Federici (2018) la cultura patriarcal ha normalizado que el trabajo reproductivo, doméstico y de cuidados sea otorgado por mujeres, por lo cual, para la sociedad no representa una "anormalidad" que la figura paterna evada sus responsabilidades o simbólicamente "aborte" a la criatura una vez nacida. 
Lo anterior se traduce en una importante sobrecarga para la mujer madre adolescente viéndose afectada sus actividades de la vida cotidiana, tal como lo expresa la categoría "El giro ocupacional: sus repercusiones psicosociales". Casanueva (2017) señala que la edad resulta ser la variable predictora de problemas de adaptación en la gestación. En ese sentido, el autor evidencia importantes consecuencias psicosociales que conlleva la etapa de crianza en aquellas mujeres que ocupan el rol de madre. A partir de la subcategoría "La privación ocupacional: relaciones socio-comunitarias en crisis", autores como Arias et al. (2011) consideran que la crianza puede impactar tanto en el desarrollo social y autónomo, como en la construcción de la identidad personal de la adolescente. Coinciden en esta valoración, Hernández \& Gentile (2018) señalando que las madres adolescentes se encuentran en situaciones complicadas e indeseadas que afectan seriamente tanto su bienestar actual como el de sus planes futuros.

Sobre esto último, la subcategoría "La interrupción educativa: como condicionante ocupacional del futuro" refleja la dificultad de llevar a cabo las labores de estudio, crianza y cuidado personal. En ese sentido, Vélez \& Figueredo (2015) evidencian una reducción en cuanto a las posibilidades de recibir una educación adecuada, así como la oportunidad del desarrollo de habilidades para garantizar el futuro económico en la población de madres adolescentes. Junto a esto, afirman que la maternidad adolescente repercute de forma negativa en el régimen ocupacional, puesto que presentan un cambio radical de cara a las ocupaciones que realizaban con anterioridad a ocupar el rol de madres. La subcategoría "El empleo: la demanda obligatoria por la (re)producción y la productividad" refleja la incompatibilidad entre las variables mencionadas. Bombino \& Quintana (2015) señalan que la autonomía que las mujeres adolescentes buscan una vez visualizándose como madres, se solapa con las ocupaciones propias de su edad, viéndose dificultado el acceso a un empleo que les permita criar y a la vez continuar con sus estudios.

Las limitaciones de este estudio se asocian al número de muestra, ya que pudiese haber enriquecido los hallazgos un número mayor de participantes. Así también, la dificultad para acceder en su totalidad a las entrevistadas de manera presencial, esta escenario hubiese aportado mayor fluidez en la interacción y el diálogo, a partir de las experiencias narradas por las participantes.

\section{Conclusiones}

La maternidad de mujeres adolescentes presenta grandes desafíos para la salud pública y las políticas sociales, sobre todo, de cuidados. En ese sentido, las dimensiones analizadas develan no sólo un desequilibrio ocupacional reflejado en la diversidad de ocupaciones que las mujeres madres adolescentes se encuentran privadas de hacer, sino también, reflejan una alienación ocupacional, en tanto se restringe la experimentación de ocupaciones significativas y enriquecedoras propias del periodo de vida, además de producirse una intensificación del trabajo remunerado y no remunerado en la experiencia cotidiana de las jóvenes madres, quienes deben reestructurar sus ocupaciones en base al cuidado y la productividad.

Por ello, los y las terapeutas ocupacionales, como promotores de la salud, pueden actuar identificando posibles factores de riesgo que afecten a la calidad de vida diaria de las mujeres madres adolescentes, teniendo en cuenta la justicia ocupacional como base, 
para prevenir o reducir la limitación o la privación que presentan respecto a su participación colectiva y en la crianza de su hijo/a.

Ante este escenario, la Terapia Ocupacional Feminista emerge como una posición epistemológica de resistencia ante las diversas manifestaciones de injusticias, marginación y alienación ocupacional que encontramos en la cotidianidad, y que experimentan y rodean al colectivo de mujeres madres adolescentes, ya que comprende que el origen y reproducción de estas injusticias, son ocasionadas en gran parte, por las desigualdades genéricas entre hombres y mujeres, los estigmas asociados a la maternidad y las condiciones precarias de viabilidad que emergen de los modelos capitalistas, patriarcales y edadistas.

\section{Agradecimientos}

Agradecemos a todas las mujeres que desinteresadamente colaboraron con sus experiencias en esta investigación.

\section{Referencias}

Allen, B., \& Waterman, H. (2019). Etapas de la adolescencia. American Academy of Pediatrics. Recuperado el 8 de octubre de 2020, de https://www.healthychildren.org/Spanish/agesstages/teen/Paginas/Stages-of-Adolescence.aspx

Amoroso, M., Bosch, A., Carrasco, C., Fernández, H., \& Moreno, N. (2003). Malabaristas de la vida: mujeres, tiempos y trabajos. Barcelona: Icaria Editorial. Grupo "Dones i Treballs” de Ca la Dona, Barcelona.

Arendt, H. (1958). La condición humana. Buenos Aires: Paidós.

Arias, E., Puntí, J., Torralbas, J., Naranjo, M. C., \& Palomino, J. (2011). Terapia ocupacional en adolescentes con retraimiento social: análisis de las áreas de desempeño ocupacional. TOG (A Coruña), 8(13), 1-15.

Bombino, Y., \& Quintana, N. (2015). Ser madre adolescente: "una experiencia bonita, pero complicada”. Estudio de casos en Barinas, 2012. Novedades en Población, (21), 78-88.

Calquín-Donoso, C., \& Yáñez-Urbina, C. (2020). Metáforas de la maternidad en un sistema de atención sanitaria de la infancia en Chile: entre la naturaleza y el capital humano. Musas, 5(2), 44-59. http://dx.doi.org/10.1344/musas2020.vol5.num2.3.

Cano, L. (2019). Asi han evolucionado las ciudades españolas: grandes capitales venidas a menos y otras en expansión. Recuperado el 8 de octubre de 2020, de https:/www.abc.es/sociedad/abci-evolucionadociudades-espanolas-grandes-capitales-venidas-menos-y-otras-expansion-201903270343_noticia.html

Casanueva, N. (2017). Adaptación psicosocial al embarazo en la adolescencia: un programa de intervención (Tesis doctoral). Escuela Universitaria de Extremadura, Espańa.

Chodorow, N. (1984). El ejercicio de la maternidad. Psicoanálisis y sociología de la maternidad y paternidad en la crianza de los hijos. Barcelona: Gedisa.

Corbin, J., \& Strauss, A. (2008). Basics of qualitative research: Techniques and procedures for developing grounded theory. Thousand Oaks: Sage Publications.

Daguerre, A., \& Nativel, C. (2006). When children become parents: welfare state responses to teenage pregnancy. Bristol: Policy Press.

Diniz, E., Garcia-Dias, A. C., Neiva-Silva, L., Nieto, C. J., \& Koller, S. H. (2012). Características familiares y apoyo percibido entre adolescentes brasileños con y sin experiencia de embarazo. Avances en Psicología Latinoamericana, 30(1), 65-80.

Dye, F., Schatz, I., Rosenberg, B., \& Coleman, S. (2000). Constant comparison method: a kaleidoscope of data. Qualitative Report, 4(1), 1-10. 
Espanha. (2020). Interrupción Voluntaria del Embarazo. Datos definitivos correspondientes al año 2018. Madrid: Ministerio de Sanidad.

Estevan-Reina, L., Rodríguez-Sánchez, R. M., Romero-González, B., Rodríguez-López, A., \& RomoSola, M. (2014). Doble presencia: un riesgo psicosocial que evidencia la desigualdad entre hombres y mujeres en la conciliación de la vida familiar y laboral. Reidocrea, 3(22), 172-179. http://dx.doi.org/10.30827/Digibug.32323.

Federici, S. (2018). El patriarcado del salario. Criticas feministas al marxismo. Madrid: Traficantes de Sueños.

Foucault, M. (2009). Defender la sociedad. Buenos Aires: Fondo de Cultura Económica.

Freixas Farré, A. F. (1995). La adquisición del género: el lugar de la educación en el desarrollo de la identidad sexual. Apuntes de Psicología, 30(1), 155-164.

Ganji, J., Emamian, M. H., Maasoumi, R., Keramat, A., \& Merghati-Khoei, E. (2018). Qualitative needs assessment: iranian parents' perspectives in sexuality education of their children. Journal of Nursing Midwifery Science, 5(4), 140-146.

García, G. (2014). Embarazo adolescente y pobreza, una relación compleja. Revista de Ciencias Sociales y Humanidades, 35(77), 13-53. http://dx.doi.org/10.28928/ri/772014/atc1/garciahernandeze.

García-Odio, A., \& González, M. (2018). Factores de riesgo asociados a embarazadas adolescentes en un área de salud. Revista Ciencia Médica, 22(3), 416-427.

Gilligan, C. (2013). Ética del cuidado. Barcelona: Cuadernos de la Fundación Victor Grifols i Lucas.

Grandón, D. (2019). Función económica de las ocupaciones feminizadas no remuneradas: una crítica desde la economía feminista. Revista Ocupación Humana, 18(2), 54-67. http://dx.doi.org/10.25214/25907816.228.

Guajardo, A. (2012). Enfoque y praxis en Terapia Ocupacional. Reflexiones desde una perspectiva de la Terapia Ocupacional critica. TOG (A Coruña), 9(5), 18-29.

Henríquez-Valencia, G. (2016). Madres adolescentes: significados y experiencias: una mujer debe poder decir sin sentirse culpable ¿Quién soy y qué quiero de la vida? (Tesis de pregrado). Universidad de Sevilla, España.

Hernández, A., \& Gentile, A. (2018). Nota de investigación: narrativas y representaciones de la maternidad en edad adolescente. Inguruak Revista Vasca de Sociología y Ciencia Politica, 64, 111-122.

Hocking, C. (2020). La justicia ocupacional como justicia social: la demanda moral de la inclusión. Journal of Occupational Science, 24(1), 29-42. http://dx.doi.org/10.1080/14427591.2017.1294016.

Hoffman, S. (1998). Teenage childbearing is not so bad after all... or is it? A review of the new literature. Family Planning Perspectives, 30(5), 236-243.

Ibarra, L. (2003). Adolescencia y maternidad: impacto psicológico en la mujer. Revista Cubana de Psicología, 20(1), 43-47.

Loredo-Abdalá, A., Vargas-Campuzano, E., Casas-Muñóz, A., González-Corona, J., \& Gutiérrez-Leyva, C. (2017). Embarazo adolescente: sus causas y repercusiones en la diada. Revista Medica del Instituto Mexicano del Seguro Social, 55(2), 223-229.

Martín-Crespo, M., \& Salamanca, A. (2007). El muestreo en la investigación cualitativa. Nure Investigación, 27, 1-4.

Morrison, R., \& Araya, L. (2018). Feminismo(s) y terapia ocupacional: preguntas y reflexiones. Revista Argentina de Terapia Ocupacional, 4(2), 60-72.

Moruno, P., \& Fernández, P. (2012). Análisis teórico de los conceptos privación, alienación y justicia ocupacional. TOG (A Coruña), 9(5), 44-325.

O’Brien, B. C., Harris, I. B., Beckman, T. J., Reed, D. A., \& Cook, D. A. (2014). Standards for reporting qualitative research: a synthesis of recommendations. Academic Medicine, 89(9), 12451251. http://dx.doi.org/10.1097/ACM.0000000000000388.

Organización Mundial de la Salud - OMS. (2018). El embarazo en la adolescencia. Recuperado el 8 de octubre de 2020, de https://www.who.int/es/news-room/fact-sheets/detail/adolescent-pregnancy.

Robles, B. (2011). La entrevista en profundidad: una técnica útil dentro del campo antropofísico. Cuicuilco, 18(52), 39-49. 
Rojas, M., \& Méndez, R. (2016). El embarazo en adolescentes: una lectura social en clave cuantitativa. Revista de la Universidad Industrial de Santander : Salud, 48(1), 81-90.

Sanz, S. (2012). Reflexiones y aprendizajes en torno a la rehabilitación basada en la comunidad. TOG ( $A$ Coruña), 9(5), 206-325.

Sibrian-Díaz, N. (2021). Ética del cuidado gestante: entre lógicas tradicionales y nuevas formas de organización en un contexto chileno. Reflexiones, 100(1), 1-21. http://dx.doi.org/10.15517/rr.v100i1.41941.

The Save the Children Fund - SCF. (2016). Every last girl: Free to live, free to learn, free from harm. Londres: The Save the Children Fund.

Timoneda, A. B. (2005). Adolescencia femenina y riesgo social: una relación invisible. Educación Social, (29), 63-78.

Valles, M. (2002). Entrevistas cualitativas. Madrid: Centro de Investigaciones Sociales

Vélez, E., \& Figueredo, E. (2015). Impacto psicosocial del embarazo en las adolescentes. Revista Educación en Valores, 1(23), 18-28.

Vidal, M. I., López, E., \& Royo, N. (2017). Visibilizando los cuidados desde una perspectiva feminista en terapia ocupacional. Revista Terapia Ocupacional Galicia, 15(27), 185-190.

Vivas, E. (2019). Mamá desobediente: una mirada feminista a la maternidad. Barcelona: Capitán Swing.

Wilcock, A., \& Townsend, E. (2000). Occupational terminology interactive dialogue. Journal of Occupational Science, 7(2), 84-86. http://dx.doi.org/10.1080/14427591.2000.9686470.

Wittig, M. (1992). El pensamiento heterosexual y otros ensayos. Barcelona: Egales.

\section{Contribución de los Autores}

Paula Mesa Molina, ha participado en el diseño de la investigación, en la recopilación de la información, análisis e interpretación de los datos, en la revisión crítica del manuscrito y finalmente, en la elaboración y aprobación del presente artículo. Juan Andrés PinoMorán, ha participado en el análisis, la interpretación de los datos, en la revisión crítica del manuscrito y finalmente, en la elaboración y aprobación del presente artículo. Pía Rodríguez-Garrido, ha participado en el análisis y revisión del material teórico y metodológico, en la revisión crítica del estudio y, finalmente, en la elaboración y aprobación del presente artículo. Todos los autores aprueban la versión final del texto.

Autor para la correspondencia

Pía Rodríguez-Garrido

e-mail: piarodriguezgarrido87@gmail.com

\section{Editor de sección}

Prof. Dr. Rodolfo Morrison 